\title{
Anticancer effects of Pimaric acid is mediated via endoplasmic reticulum stress, caspase-dependent apoptosis, cell cycle arrest, and inhibition of cell migration in human ovarian cancer cells
}

\author{
Wang Li1, Gao Xuemei', Zhu Yilin1, Wang Han'1, Hu Yajun', He Yi and Zhu Zhongxiang²凶 \\ 1Department of Obstetrics and Gynecology, Wuhan No.1 Hospital, Wuhan, 430000, China; 2Department of Radiology, Wuhan PuRen Hospital, \\ Wuhan, 430000, China
}

Pimaric acid is a naturally occurring resin and has been found to perform many pharmacological activities including, anticancer activity. However, the role of Pimaric acid in ovarian cancer is still not known. This investigation aimed to evaluate the anticancer effects of Pimaric acid and its molecular mechanism in human ovarian cancer cells. MTT assay was used to examine cell viability. Cell morphology was determined through phase contrast microscopy. DAPI staining and TUNEL assay were performed for apoptotic study. Examination of cell cycle phase distribution was carried out through flow cytometry. In vitro wound healing assay was used for cell migration determination. Pimaric acid induced cytotoxicity in human ovarian cancer cells (PA-1) in a dose-dependent manner without causing too much cytotoxicity in human ovarian epithelial cells (T1074). Cell morphology in treated cancer cells showed significant changes compared to untreated controls. Furthermore, it was observed that the cytotoxic effects of Pimaric acid were apoptosis-mediated and caspase-dependent cascade. Western blotting analysis showed that the expression of apoptosisassociated proteins like BAX, p-53 and caspase- 3 was enhanced and BCL-2 expression was diminished. The induction of cytotoxicity was mediated via endoplasmic reticulum stress through expressions of related proteins which showed a tremendous increase in p-PERK, PERK, AT-4, CHOP and IRE-1 levels after treatment. Cell cycle analysis through cytometry showed significant results as it revealed G2/M phase cell cycle arrest. Furthermore, the in vitro wound healing assay showed specific antimigratory effects of Pimaric acid on PA-1 cells. In conclusion it can be assumed that Pimaric acid may act as a potential anticancer agent against ovarian carcinoma, however further investigations are required to validate this initial claim.

Keywords: Pimaric acid, ovarian cancer, apoptosis, cell cycle, caspase Received: 10 November, 2021; revised: 29 December, 2021; accepted: 30 December, 2021; available on-line: 02 March, 2022

$凶$ e-mail: zhuzhongxiang20@gmail.com

Abbreviations: OC, Ovarian cancer; DAPI, 4,6-diamidino-2-phenylindole; BCA, bicinchoninic acid; Bcl-2, B-cell lymphoma 2; PERK, Protein Kinase RNA-Like ER Kinase; CHOP, C/EBP Homologous Protein

\section{INTRODUCTION}

Ovarian cancer (OC) is a lethal malignancy affecting large numbers of women across the globe (Miree et al., 2021). Due to its asymptotic behaviour, the maximum number of patients is diagnosed at later disease stages.
Only $2.5 \%$ of women are diagnosed of OC worldwide, but the death rate is $5 \%$. Poor prognosis results show decreased 5-year overall survival (OC) rate, which stands at 25\% to 30\% (Fleming et al., 2011). In 2018 alone, around 22000 new patients and 14000 deaths due to OC were expected in United States.

Ovarian cancer is divided into three main subtypes: epithelial OC, germ cell OC and sex cord-stromal OC (Miree et al., 2021). Various factors contribute to the etiology of this disease including biological, physical, chemical, genetic, immune factors, carcinogenic factors, and poor lifestyle choices. Intraperitoneal chemotherapy has revealed significant improvements in later stages of epithelial OC. Still, in 2012, only less than $50 \%$ of the patients were treated with intraperitoneal chemotherapy due to a higher number of side effects (Reid et al., 2017; Kurnit et al., 2021).

OC has a very high relapse rate and develops high chemotherapy resistance, resulting in high mortality and poor prognosis (Feng et al., 2021). An increased overall survival rate was reported after secondary surgery in case of relapsing. Thus it may be considered for the patients who have a disease-free time interval of half a year (Fleming et al., 2011; Al Rawahi et al., 2013) (Tangjitgamol et al., 2013). Lethality of OC gets amplified mainly of poor prognosis, diagnosis at later stages, drug resistance and side effects of chemotherapy. Therefore there is a pressing need to overcome the shortcomings of conventional chemotherapeutic agents and move to novel and effective ones.

Apoptosis plays a crucial role in maintaining the balance between cell division and death, mandatory for the normal physiology of cells. Hence, downregulation of this process results in leading disorders, including cancer. Moreover, minimal inflammation is witnessed during apoptotic cell death, which has attracted strong attention in developing chemo-preventives targeting apoptosis. Apoptosis is modulated by intra and extra-cellular signalling transduction known as intrinsic and extrinsic apoptosis, respectively (Putcha et al., 2002). Death receptor proteins stimulates extrinsic pathway, while intrinsic pathway is mostly mitochondrial mediated. Intrinsic apoptosis begins with the insertion of $\mathrm{Bax} / \mathrm{Bak}$ proteins into the mitochondrial membrane, which stimulates the release of cytochrome-c into cytosol (Pena-Blanco and Garcia-Saez, 2018). Cytochrome-c initiates a number of proapoptotic interactions within cytosol which ultimately leads to apoptotic cell death. One of the main functions performed by cytochrome- $c$ is activation of different caspases (Jiang \& Wang, 2004). So, anticancer molecules are always tested in their capacity to activate apoptotic pathways in cancer kills. 
Due to their amazing structural diversity and potential bioactivity profiles, natural products, continuously influence the drug discovery researchers (Tang et al., 2010). The carboxylic acid group shows a key role in bio-systems and drug design. Over 450 drugs containing carboxylic acid functional group are being used for humans (Kuo et al., 2010). Pimaric acid - a naturally occurring resin carboxylic acid - has revealed several pharmacological activities, including cancer ( $\mathrm{Zi} \&$ Simoneau, 2005). Treatment of brain tumor cells with Pimaric acid has been reported to significantly kill the tumour cells (Hoa et al., 2009). Herein, the current study was performed to examine the anticancer effects of Pimaric acid in human ovarian cancer cells.

\section{METHODS}

\section{Cell culture}

Cell lines T1074 (human ovarian normal epithelial cells) and PA-1 (human ovarian cancer cells) were purchased from ATCC. Cells were grown in culture for $24 \mathrm{~h}$ in RPMI-1640 medium mixed with 10\% FBS and supplemented with antibiotic solution $(100 \mathrm{U} / \mathrm{ml})$. Cells were grown under normal conditions $\left(5 \% \mathrm{CO}_{2}\right.$ with $95 \%$ humidified air). Cells were either left untreated or treated with different concentrations of Pimaric acid. Pimaric acid (CAS 127-27-5) was obtained from Santa Cruz.

\section{Reagents}

Antibodies against p-PERK (Cat no. 3179S), PERK (Cat no. 3192S), AT-4 (Cat no. 11815S), CHOP (Cat no. 2895S), IRE-1 (Cat no. 3294S), Cyclin-B1 (Cat no. 4138S) and $\beta$-actin (Cat no. 4967S) were purchased from Cell Signalling Technology, MA, United States. All other chemicals used were acquired from Sigma-Aldrich (St. Louis, MO, USA).

MTT assay. The cytotoxicity of Pimaric acid was determined through MT'T assay. Both the cell lines T1074 and PA-1 $\left(1 \times 10^{3}\right.$ cells) were plated using 96-well plates and subjected to incubation for $12 \mathrm{~h}$ in a $5 \% \mathrm{CO}_{2}$ incubator. Incubation was followed by treatment with different doses of Pimaric acid (0, 2.5, 5, 10, 20, 40, 80, 160 and $320 \mu \mathrm{M})$. After treatment, cells were further incubated for $48 \mathrm{~h}$ in a $5 \% \mathrm{CO}_{2}$ incubator at $37^{\circ} \mathrm{C}$. At the end of Pimaric acid treatment, MTT (Sigma) stock solution of $5 \mathrm{mg} / \mathrm{mL}$ concentration and volume $100 \mu \mathrm{L}$ was supplemented to cells with $4 \mathrm{~h}$ of incubation. The formazan crystals then produced are dissolved using DMSO, and thereafter, absorbance was measured at $580 \mathrm{~nm}$ using OPTImax microplate reader (Molecular Devices, San Jose, California, USA).

Cell morphology determination by phase-contrast microscopy. Cell morphological changes of Pimaric acid-treated cells were determined by phase contrast microscopy. Briefly, $4 \times 10^{4} \mathrm{PA}-1$ human ovarian cancer cells were incubated for $48 \mathrm{~h}$ with varying concentrations of Pimaric acid $(0,5,10$ and $20 \mu \mathrm{M})$ in 6-well plates. Afterward, RMPI-1640 medium was discarded and cells were washed using phosphate buffered saline (PBS). The morphological differences between cells were visible. Phase contrast inverted microscope (Leica DMI 3000B, Germany) was used (magnification $\times 200$ ) to detect the morphological changes in apoptotic cells (Syed et al., 2013).

Cell apoptosis determination. PA-1 human ovarian cancer cells were seeded at a concentration of $3 \times 10^{3}$ cells per well in 96-well plates. Seeding was followed by treatment with Pimaric acid at varying concentrations $(0,5,10$ and $20 \mu \mathrm{M})$. Cells were then incubated at $37^{\circ} \mathrm{C}$ for $24 \mathrm{~h}$. After incubation of treated cells, 4',6-diamidino-2-phenylindole staining (DAPI) was performed. The treated cancerous cells were then washed in PBS and fixed using 10\% formaldehyde. Finally, DAPI-stained cells were examined under fluorescence microscope.

To determine the extent of apoptotic cells TUNEL assay was used. PA-1 human ovarian cancer cells were seeded with different concentrations of Pimaric acid using 6-well plates with coverslips to protect from any contamination for $12 \mathrm{~h}$. Seeded cells were washed and fixed with PBS with acetone-methanol (1:1), respectively, for 15 min. DNA damage by apoptosis induction was evaluated through terminal deoxynucleotidyl transferase-UTP mediated nick end labelling using in Situ Cell Death Detection Kit (Roche Diagnostics, Mannheim, Germany) according to the manufacturer's protocol.

Cell cycle analysis. For cell phase distribution of PA-1 human ovarian cancer cells, cells were harvested and PBSwashed. Washing was followed by treatment with varying doses of Pimaric acid (control, 5, 10 and $20 \mu \mathrm{M}$ ). These treated cells were then subjected to further washing and fixation $(70 \%$ ethanol). After that, cells were suspended in $250 \mu \mathrm{g} / \mathrm{ml}$ of RNase1 and $50 \mu \mathrm{l} / \mathrm{ml}$ of propidium iodide (PI) solution and incubated for one hour at room temperature. Finally, grouping was performed such that 10000 cells were arranged in each group and examination was done through fluorescence-activated cell sorting using FACSCalibur flow cytometer.

In vitro wound healing assay. PA-1 human ovarian cancer cells at a density of $3 \times 10^{4}$ were placed in 6 -well plates using RPMI-1640 medium. Using a $10-\mu \mathrm{L}$ pipette tip, scratch or wound was produced in each well, and then cells were left untouched till they reached 90\% confluence of growth. RMPI-1640 medium was decanted and cells were washed with PBS. Cultures cells were treated with Pimaric acid at the varying concentrations (control, 5, 10 and $20 \mu \mathrm{M}$ ). Six randomly selected fields were captured (magnification $\times 200$ ) using microscopy before and after treatment $(24 \mathrm{~h})$.

Western blotting analysis. Foe western blotting analysis, cells were lysed using RIPA buffer. Estimation of protein lysate was performed through bicinchoninic acid (BCA) assay. Protein samples were loaded on SDSPAGE and were transferred to PVDF membrane. Next, these membranes were subjected to primary antibody treatment for $24 \mathrm{~h}$ at $4^{\circ} \mathrm{C}$. Primary antibody treatment was followed by HRP-conjugated secondary antibody treatment at room temperature for $1 \mathrm{~h}$. Chemi-luminescence reagent (Beyotime Institute of Biotechnology, Jiangsu, China) was used to develop and visualize protein bands.

Statistics. All the data were presented as mean \pm standard deviation (S.D.) from at least three independent experiments. Statistically, significant difference was taken as $p<0.05$. Differences among the groups were investigated through one-way ANOVA with Graphpad Prism 7 software.

\section{RESULTS}

\section{Effects of Pimaric acid on cell viability}

Cell viability effects on T1074 and PA-1 by Pimaric acid (Fig. 1A) were analysed through MT'T assay. Cell viability was determined after treatment with different dos- 


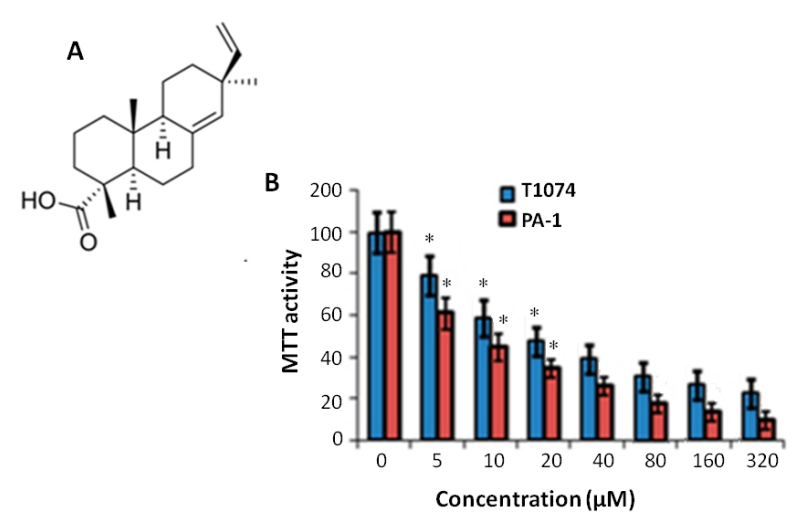

Figure 1. (A) Chemical structure of Pimaric acid. (B) Effect of Pimaric acid on the proliferation of T1074 and PA-1 cells were evaluated through MTT assay.

The experiments were performed three times and in triplicates, ${ }^{*} p<0.05$.

es of Pimaric acid. The results depicted that the viability of PA-1 cells decreased significantly with increasing drug concentrations (Fig. 1B). The cell cytotoxicity induced in normal T1074 ovarian epithelial cells was much lower than the cancer cells indicating its selective cytotoxicity.

\section{Pimaric acid induced significant morphological alterations in PA-1 ovarian cancer cells}

Morphological changes were determined by using phase contrast microscopy after exposure to varying doses of Pimaric acid (control, 5, 10 and $20 \mu \mathrm{M}$ ). Control cells showed no significant changes in the cell morphology and maintained their originality. But the morphology of treated PA-1 cells showed significant changes including cell shrinkage, membrane blebbing, cell rounding, and dropping contact with nearby cells (Fig. 2).
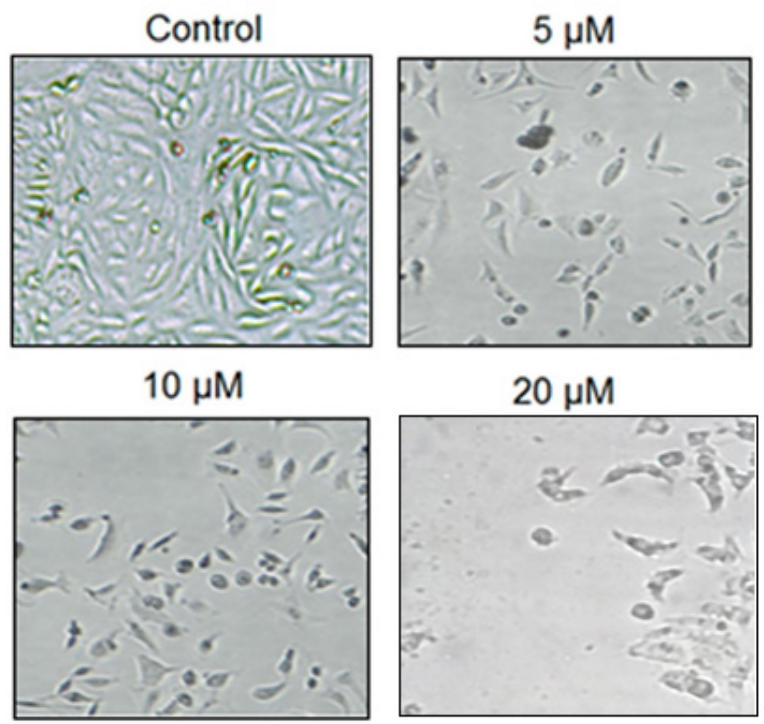

Figure 2. The effects of Pimaric acid on morphology of PA-1 human ovarian cancer cells.

Pimaric acid-treated cells showed significant morphological changes like cell shrinkage, membrane blebbing cell rounding, and dropping contact with nearby cells. The experiment was performed three times and in triplicates. The above picture shows

\section{Induction of caspase-dependent apoptosis by Pimaric} acid in PA-1 cells

Estimation of apoptosis in PA-1 cells was performed via DAPI staining and TUNEL assay. DAPI staining revealed significant changes in the morphology of the cells after Pimaric acid exposure (control, 5, 10 and $20 \mu \mathrm{M}$ ). Fluorescence microscopy showed membrane blebbing and the onset of formation of apoptotic crops after treatment. No such effect was observed
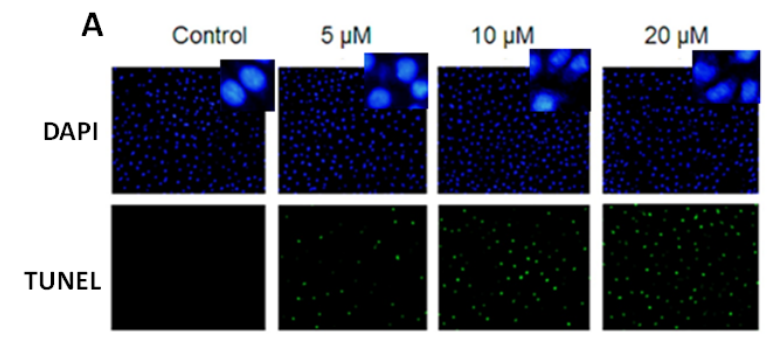

Q Bcl-2

Bax

B

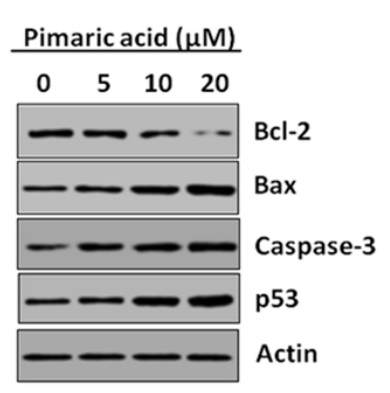

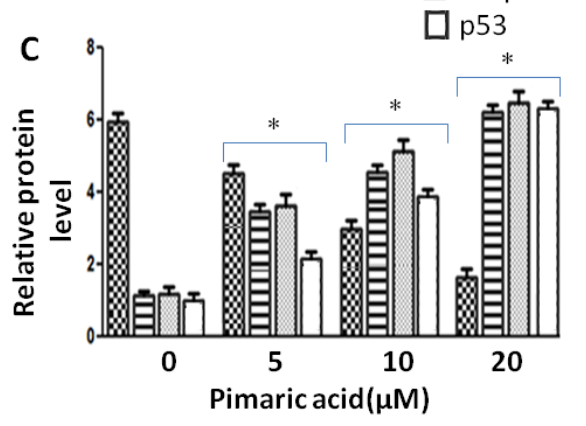

Figure 3. Title for the whole figure

(A) DAPI staining (blue) and TUNEL assay. Apoptosis determination at varying concentrations of Pimaric acid (control, 5, 10 and $20 \mu M$ ). As indicated treated cells showed apoptotic crop formation and membrane blebbing. TUNEL-positive apoptotic cells were detected by localized FITC-fluorescence (green). (B) Effect of Pimaric acid assessed by western blotting on the expression of apoptosis related proteins including BCL-2, BAX, p-53 and caspase-3. The results depicted that expression of BAX, p-53 and caspase-3 increased and that of BCL-2 decreased post Pimaric acid treatment. Actin was taken as loading control. (C) Shows densitometry analysis of western blots. The experiments were performed three times and in triplicates. 
A

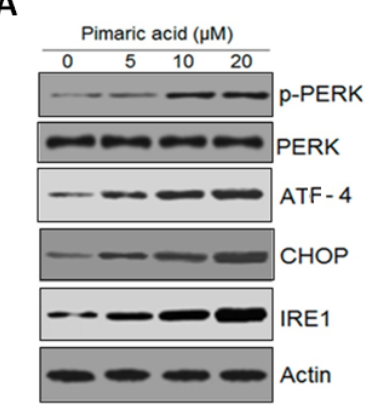

C

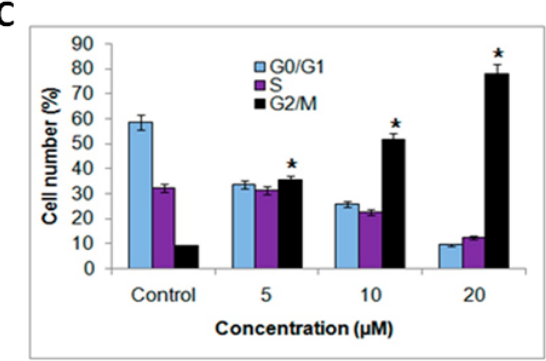

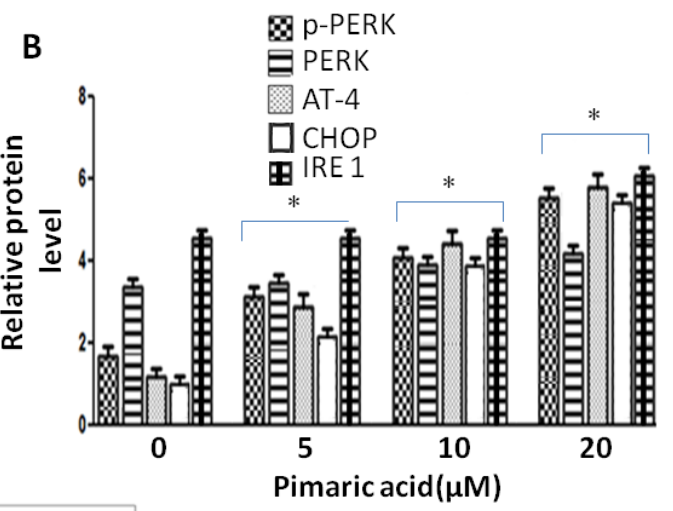

D

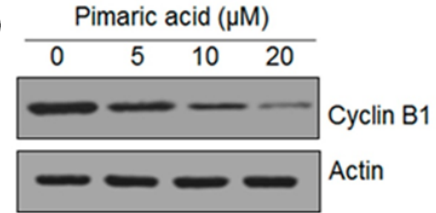

Figure 4. One title for the whole figure

(A) Effect of Pimaric acid assessed by western blotting on the expression of proteins associated with endoplasmic reticulum stress including p-PERK, PERK, AT-4, CHOP and IRE-1, with Actin as loading control. The results indicated increased levels of expressions of $p$-PERK, PERK, AT-4, CHOP and IRE-1 in PA-1 cells post Pimaric acid treatment. (B) Shows densitometry analysis of western blots. (C) Cell cycle phase distribution after treatment with varying doses of Pimaric acid (control, 5, 10 and $20 \mu \mathrm{M}$ ). With increasing molecule concentrations the number of G2/M phase cells increased significantly as indicated. The value is shown as mean \pm S.D. $\left({ }^{*} p<0.05\right)$. (D) Western blotting analysis showing the expressions of cell cycle related protein Cyclin-B1 in Pimaric acid treated (0, 5, 10 and $20 \mu \mathrm{M})$ PA-1 cells. The figure depicts decreasing expressions of Cyclin-B1 in PA-1 cells on increasing the concentrations of Pimaric acid. The experiments were performed three times and in triplicates.

in untreated cells. Apoptotic studies were not carried out in case of normal cells (T1074 ovarian epithelial cells). Furthermore, it was revealed that the number of apoptotic cells increased with increasing dose of Pimaric acid. Next, TUNEL assay revealed that the apoptosis induction by Pimaric acid was mediated via DNA damage as the frequency of stained cells increased with increasing molecule concentration (Fig. 3A). Furthermore, western blotting analysis was performed to evaluate the effects of Pimaric acid on the expressions of apoptosis-related proteins. Results showed that the expression of BAX, p-53 and caspase-3 increased post Pimaric acid exposure, however expression level of BCL-2 decreased significantly (Fig. 3B), revealing induction of caspase-dependent apoptosis.

\section{Antiproliferative effects of Pimaric acid via endoplasmic reticulum stress and cell cycle arrest}

The expression of endoplasmic reticulum (ER) stress related proteins in treated PA-1 human ovarian cancer cells were evaluated via western blotting analysis, depicting that the levels of ER stress related proteins (p-PERK, PERK, AT-4, CHOP and IRE-1) increased significantly on increasing the molecule concentration (control, 5, 10 and $20 \mu \mathrm{M}$ ), suggesting that the antiproliferative effects of Pimaric acid on PA-1 cells are clearly induced via induction of ER stress (Fig. 4A).

After exposure of PA-1 human ovarian cancer cells with the test molecule at different concentrations (control, 5, 10 and $20 \mu \mathrm{M}$ ), cell cycle analysis was performed via fluorescence-activated cell sorting using flow cytometer. The results showed that the number of $\mathrm{S}$ phase and G0/G1 phase cells decreased with increased dose concentrations of Pimaric acid. There was a tremendous increase in the number of G2/M phase cells, suggestive of cell cycle arrest at this phase of the cell cycle distribution (Fig. 4B). It was further validated via western blotting analysis which revealed significant decrease in the expression of Cyclin-B1 protein (Fig. 4C).

\section{Inhibition of cell migration by Pimaric acid on PA-1 human ovarian cancer cells}

The impact on PA-1 cell migration by Pimaric acid was studied through in vitro wound healing assay. Cells were treated with different doses of Pimaric acid after an in vitro wound was scratched in each well. Photographs were captured after $0 \mathrm{~h}$ and $24 \mathrm{~h}$ of exposure at $0 \mu \mathrm{M}$ (untreated control) and $10 \mu \mathrm{M}$ of Pimaric acid concentration. The pictures suggested that the number of migrated cells was reduced compared to untreated controls $(0 \mu \mathrm{M})$ after $24 \mathrm{~h}$ of exposure (Fig. 5).

\section{DISCUSSION}

There is an alarming increase in the incidence rate of ovarian cancer resulting in high mortality worldwide. Poor prognosis, diagnosis at later-stage, high relapse, chemotherapy resistance and chemo-side effects are the major causes of mortality of this malignancy. To overcome these drawbacks of the conventional chemotherapy, we need to switch to novel and efficient methods, chemotherapeutic agents and explore different target pathways to curb OC (Rikova et al., 2007; Bergethon et al., 2012; Siegel et al., 2020). Endoplasmic reticulum response (ERR) plays a key role in regulating cell apoptosis, cell proliferation and cell autophagy in different human cancers. Its association is perhaps found in large numbers of biological systems. When there is an increase 

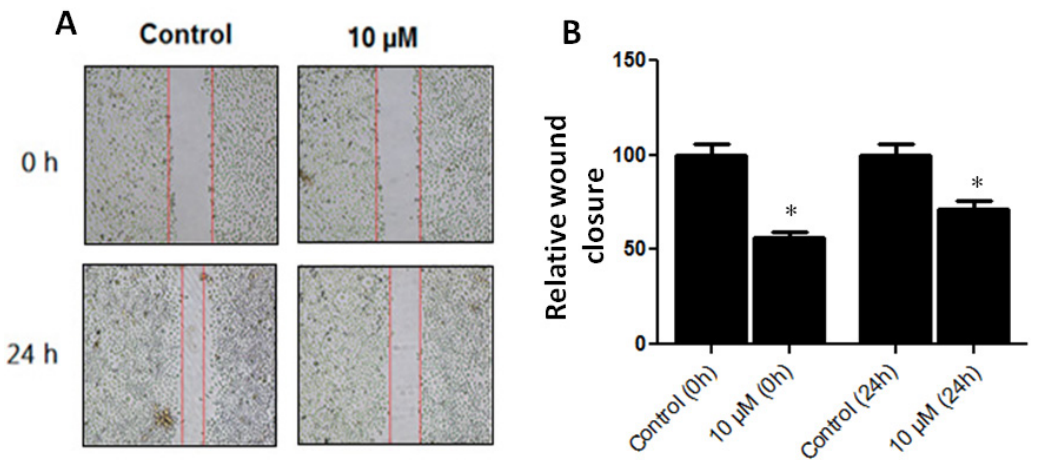

Figure 5. In vitro wound healing assay for cell migration determination.

(A) The $10 \mu \mathrm{M}$ dose of Pimaric acid led to significant cell migration inhibition in PA-1 cells. (B) Shows quantification of in vitro wound healing assay. As shown in the figure, Pimaric acid significantly inhibited cell migration by $25 \%$. The experiment was performed three times and in triplicates

in the accumulation of unfolded proteins within a cell, ER releases large quantity of Bip proteins, enhancing and amplifying protein folding, reducing the amount of unfolded proteins and restoring ER function (Shaw et al., 2014). Herein, we testified the activity of Pimaric acid on cell proliferation of ovarian cancer cells via ER stress, caspase-dependent apoptosis, cell cycle arrest and inhibition of cell migration. Cell viability was determined by MT'T assay and the results revealed that the cytotoxic effects were significant on PA-1 cells as compared to T1074 cells. It also revealed that the cytotoxic effect of the test molecule were dose-dependent. Similar type of inhibitory function of Pimaric acid has been reported in brain tumor cells (Hoa et al., 2009). Furthermore, cell morphological changes were determined by phase-contrast microscopy, which showed that control cells retained their original identity in contrast with treated cells which showed significant morphological changes like cell shrinkage, membrane blebbing, cell rounding, and dropping contact with nearby cells. Next, DAPI staining and TUNEL assays were performed to assess whether the cytotoxic effects were due to apoptosis induction. Both the assays showed an increase in the number of apoptotic cells after treatment with Pimaric acid, showing membrane blebbing with formation of apoptotic crops. It was also unveiled that the number of apoptotic cells increases with increasing dose concentrations. Furthermore, the expressions of apoptosis-related proteins were altered by the exposure of target cells to the test molecule, and the expression of BAX, p-53 and caspase- 3 was increased as compared to the BCL-2 expression which was suppressed. It was also observed through western blotting analysis that whether the apoptosis was caspase and ER stress mediated the results showed tremendous increase in the expressions of p-PERK, PERK, AT-4, CHOP and IRE-1, indicating ER stress. Activation of IRE1-JNKCHOP signalling also been reported by kaempferol in gastric cancer cells (Kim et al., 2018). Afterwards, cell cycle analysis was performed after treatment with Pimaric acid. The results suggested a tremendous increase in the number of $\mathrm{G} 2 / \mathrm{M}$ phase cells compared to the $\mathrm{S}$ phase and G0/G1, clearly indicating that Pimaric acid induced G2/M phase cell cycle arrest. Cell cycle arrest has been reported by levopimaric acid in human hepatoma cells (Qi et al., 2018). Western blotting analysis also showed that the expression of Cyclin-B1 diminished after increasing the molecule concentrations. Finally, cell migration results depicted that the cell migration of PA-1 human ovarian cancer cells was significantly suppressed with increasing doses of the test molecule. The above results indicate that Pimaric acid is a potential anticancer agent against human ovarian cancer cells and merits further investigations.

\section{CONCLUSIONS}

In conclusion, through all the above assays and results it is evident that Pimaric acid is a potential anticancer agent against PA-1 human ovarian cancer cells. The results revealed that Pimaric acid induces antiproliferative effects in human ovarian cancer cells via ER stress, caspase-dependent apoptosis, cell cycle arrest, and inhibition of cell migration.

\section{Conflict of interest}

The authors declare that there is no conflict of interest to indicate.

\section{REFERENCES}

Al Rawahi T, Lopes AD, Bristow RE, Bryant A, Elattar A, Chattopadhyay S, Galaal K (2013) Surgical cytoreduction for recurrent epithelial ovarian cancer. Cochrane Database Syst Rev 2013: CD008765. https://doi.org/10.1002/14651858.CD008765.pub3

Bergethon $\mathrm{K}$, Shaw AT, Ou SH, Katayama R, Lovly CM, McDonald NT, Massion PP, Siwak-Tapp C, Gonzalez, A, Fang R, Mark EJ, Batten JM, Chen H, Wilner KD, Kwak EL, Clark JW, Carbone DP, Ji H, Engelman JA, Mino-Kenudson M, Pao W, Iafrate AJ (2012) ROS1 rearrangements define a unique molecular class of lung cancers. J Clin Oncol 30: 863-870. https://doi.org/10.1200/ JCO.2011.35.6345

Feng LY, Yan BB, Huang YZ, Li L (2021) Abnormal methylation characteristics predict chemoresistance and poor prognosis in advanced high-grade serous ovarian cancer. Clin Epigenetics 13: 141. https://doi.org/10.1186/s13148-021-01133-2

Fleming ND, Cass I, Walsh CS, Karlan BY, Li AJ (2011) CA125 surveillance increases optimal resectability at secondary cytoreductive surgery for recurrent epithelial ovarian cancer. Gynecol Oncol 121: 249-252. https://doi.org/10.1016/j.ygyno.2011.01.014

Hoa N, Myers MP, Douglass TG, Zhang JG, Delgado C, Driggers L, Callahan LL, VanDeusen G, Pham JT, Bhakta N, Ge L, Jadus MR (2009) Molecular mechanisms of paraptosis induction: implications for a non-genetically modified tumor vaccine. PloS One 4: e4631. https://doi.org/10.1371/journal.pone.0004631

Jiang X, Wang X (2004) Cytochrome C-mediated apoptosis. Annu Rev Biochem 73: 87-106. https://doi.org/10.1146/annurev.biochem.73.011303.073706

Kim TW, Lee SY, Kim M, Cheon C, Ko SG (2018) Kaempferol induces autophagic cell death via IRE1-JNK-CHOP pathway and inhibition of G9a in gastric cancer cells. Cell Death Dis 9: 875. https://doi.org/10.1038/s41419-018-0930-1

Kuo YF, Su YZ, Tseng YH, Wang SY, Wang HM, Chueh PJ (2010) Flavokawain B, a novel chalcone from Alpinia pricei Hayata with potent apoptotic activity: Involvement of ROS and GADD153 upstream of mitochondria-dependent apoptosis in HCT116 cells. Free 
Radic Biol Med 49: 214-226. https://doi.org/10.1016/j.freeradbiomed.2010.04.005

Kurnit KC, Fleming GF, Lengyel E (2021). Updates and new options in advanced epithelial ovarian cancer treatment. Obstet Gynecol 137: 108-121. https://doi.org/10.1097/AOG.0000000000004173

Miree O, Srivastava SK, Dasgupta S, Singh S, Rocconi R, Singh AP. (2021) Current and futuristic roadmap of ovarian cancer management: An overview. Adv Exp Med Biol 1330: 1-19. https://doi. org/10.1007/978-3-030-73359-9_1

Pena-Blanco A, Garcia-Saez AJ (2018) Bax, Bak and beyond - mitochondrial performance in apoptosis. FEBS J 285: 416-431. https:// doi.org/10.1111/febs.14186

Putcha GV, Harris CA, Moulder KL, Easton RM, Thompson CB, Johnson EM Jr. (2002). Intrinsic and extrinsic pathway signaling during neuronal apoptosis: Lessons from the analysis of mutant mice. J Cell Biol 157: 441-453. https://doi.org/10.1083/ jcb.200110108

Qi Z, Wang C, Jiang J, Wu C (2018) Novel C15 triene triazole, D-A derivatives anti-HepG2, and as HDAC2 inhibitors: A synergy study. Int J Mol Sci 19. https://doi.org/10.3390/ijms19103184

Reid BM, Permuth JB, Sellers TA (2017) Epidemiology of ovarian cancer: a review. Cancer Biol Med 14: 9-32. https://doi.org/10.20892/j. issn.2095-3941.2016.0084

Rikova K, Guo A, Zeng Q, Possemato A, Yu J, Haack H, Nardone J, Lee K, Reeves C, Li Y, Hu Y, Tan Z, Stokes M, Sullivan L, Mitchell J, Wetzel R, Macneill J, Ren JM, Yuan J, Bakalarski CE, Villen J, Kornhauser JM, Smith B, Li D, Zhou X, Gygi SP, Gu TL, Polakiewicz RD, Rush J, Comb MJ (2007) Global survey of phosphoty- rosine signaling identifies oncogenic kinases in lung cancer. Cell 131: 1190-1203. https://doi.org/10.1016/j.cell.2007.11.025

Shaw AT, Ou SH, Bang YJ, Camidge DR, Solomon BJ, Salgia R, Riely GJ, Varella-Garcia M, Shapiro GI, Costa DB, Doebele RC, Le LP, Zheng Z, Tan W, Stephenson P, Shreeve SM, Tye LM, Christensen JG, Wilner KD, Clark JW, Iafrate AJ (2014) Crizotinib in ROS1-rearranged non-small-cell lung cancer. N Engl J Med 371: 1963-1971. https://doi.org/10.1056/NEJMoa1406766

Siegel RL, Miller KD, Jemal A. (2020) Cancer statistics, 2020. CA Cancer J Clin 70: 7-30. https://doi.org/10.3322/caac.21590

Syed AR, Wahab AN, Abd Malek SN (2013) In vitro morphological assessment of apoptosis induced by antiproliferative constituents from the Rhizomes of Curcuma zedoaria. Evid Based Complement Alternat Med 2013: 257108. https://doi.org/10.1155/2013/257108

Tang Y, Li X, Liu Z, Simoneau AR, Xie J, Zi X (2010) Flavokawain B, a kava chalcone, induces apoptosis via up-regulation of death-receptor 5 and Bim expression in androgen receptor negative, hormonal refractory prostate cancer cell lines and reduces tumor growth. Int $J$ Cancer 127: 1758-1768. https://doi.org/10.1002/ijc.25210

Tangjitgamol S, Manusirivithaya S, Laopaiboon M, Lumbiganon P, Bryant A (2013) Interval debulking surgery for advanced epithelial ovarian cancer. Cochrane Database Syst Rev 4: CD006014. https://doi. org/10.1002/14651858.CD006014.pub6

Zi X, Simoneau AR (2005) Flavokawain A, a novel chalcone from kava extract, induces apoptosis in bladder cancer cells by involvement of Bax protein-dependent and mitochondria-dependent apoptotic pathway and suppresses tumor growth in mice. Cancer Res 65: 3479-3486. https://doi.org/10.1158/0008-5472.CAN-04-3803 